Supporting Information for

\title{
Backbone Assignments in Solid-State Proteins using J-Based 3D Heteronuclear Correlation Spectroscopy
}

\author{
Lingling Chen, ${ }^{\dagger}$ J. Michael Kaiser, ${ }^{\dagger}$ Tatyana Polenova, ${ }^{\#}$ Jun Yang, ${ }^{\#}$ Chad M. Rienstra ${ }^{\ddagger}$ and Leonard J. Mueller, ${ }^{*}+$
}

\footnotetext{
${ }^{\dagger}$ Department of Chemistry, University of California, Riverside, California 92521,

${ }^{*}$ Department of Chemistry and Biochemistry, University of Delaware, Newark, Delaware 19716, and

${ }^{\ddagger}$ Department of Chemistry, University of Illinois at Urbana-Champaign, Urbana, IL 61801
}

Complete Phase Cycles and Off-Resonance Compensation for 3D J - MAS Experiments: $S 2$

$T_{2}$ vs. MAS Rate and Decoupling Power: S4

1D traces from 3D Spectra and Anticipated Upper Size Limit for Protein Correlation: S5

NCOCA Spectrum of Reassembled Thioredoxin at 14.1 T : S6

NCOCA and NCACO Spectrum of GB1 at $14.1 T$ :

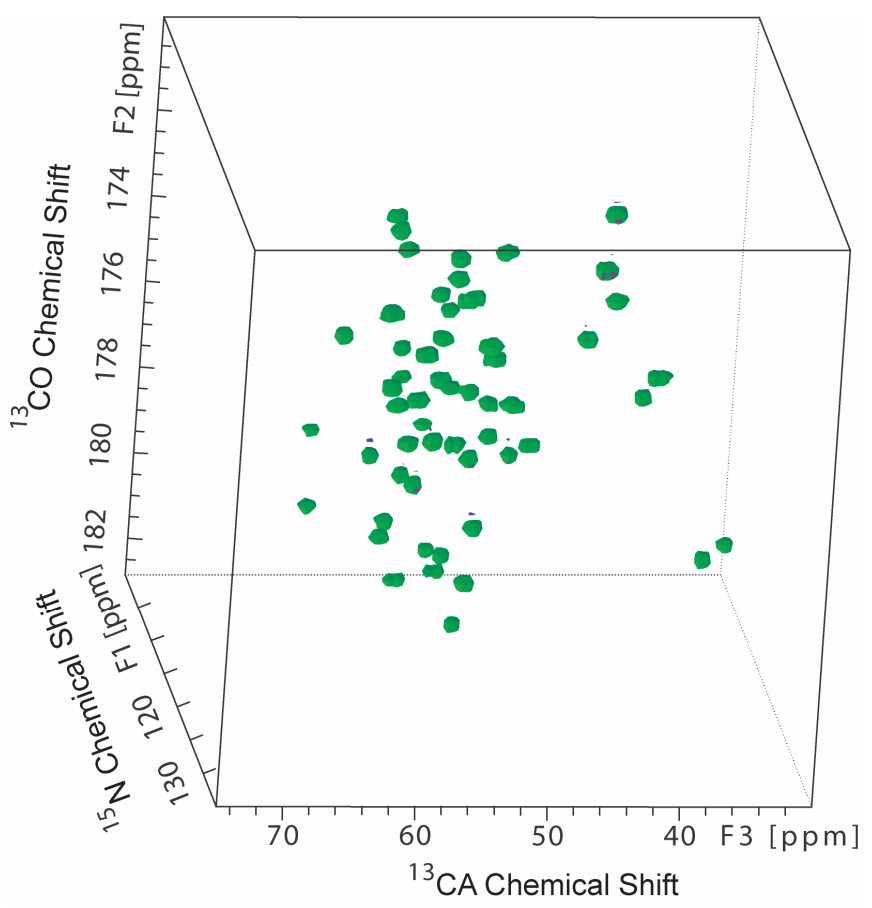


ja073498e_SI_0807.pdf

\section{Complete Phase Cycles and Off-Resonance Compensation for 3D J - MAS Experiments}

\section{NCACO and NCOCA}

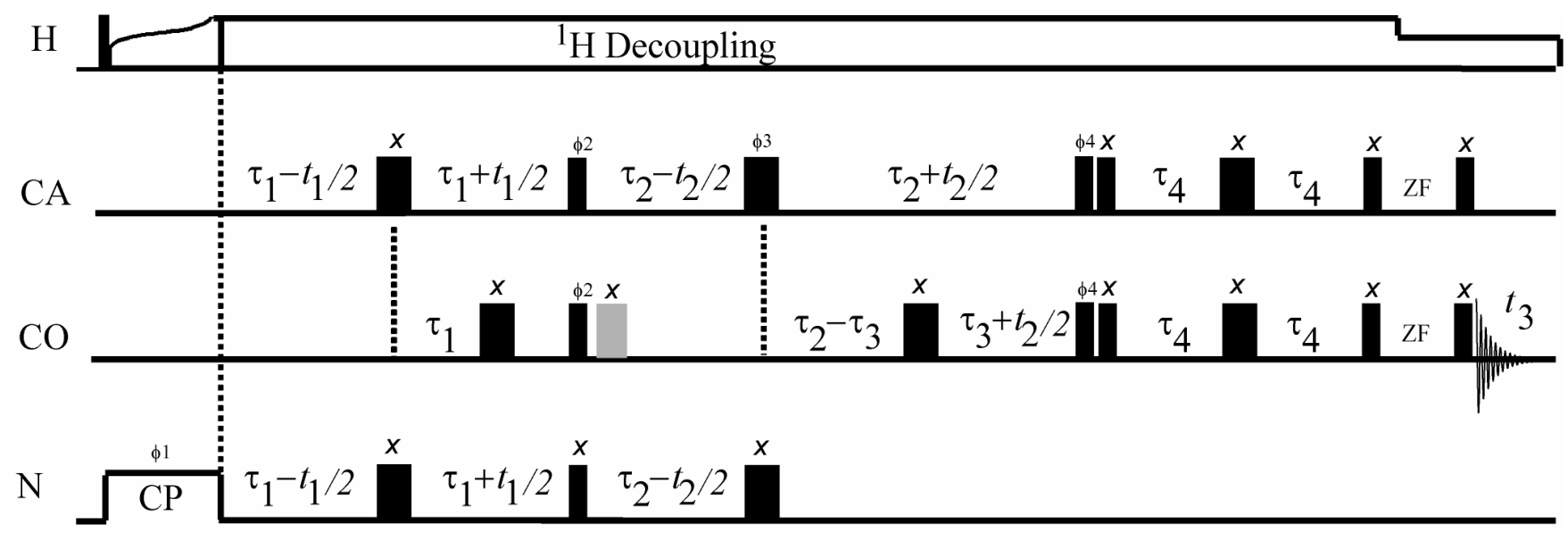

Off-Resonance Compensation: Although we did not find off-resonance effects to be significant under our experimental conditions, they could in general lead to phase errors in the indirect dimensions. In such cases, an additional $\pi$ pulse on the passive spin (shown as the grey, shaded pulse) can be incorporated to refocus off-resonance effects.

Phase Cycle: We find that a minimal phase cycle is needed. The ${ }^{15} \mathrm{~N}$ spin lock is cycled for $+/-1$ and the three ${ }^{13} \mathrm{C}$ pulses $\left(\phi_{2}\right.$, $\left.\phi_{3}, \phi_{4}\right)$ are independently phase cycled together for zero and double quantum selection. In the table below the phase of the rf is given as integer multiples of $90^{\circ}$ (i.e., $0=$ "x", $1=$ "y", $2=$ "-x", $3=$ "-y") and the list repeats once the end of the line has been reached.

3D pure phase spectra are obtained using the method of States (States, D. J.; Haberkorn, R. A.; Ruben, D. J. J. Magn. Reson. 1982, 48, 286-292) by incrementing $\phi_{1}$ by $90^{\circ}$ and $\phi_{2}$ by $90^{\circ}$ for $t_{1}$ and $t_{2}$, respectively.

\begin{tabular}{|c|l|}
\hline Phase & Cycle \\
\hline$\phi_{1}$ & 0,2 \\
$\phi_{2}$ & $0,0,2,2$ \\
$\phi_{3}$ & $0,0,2,2$ \\
$\phi_{4}$ & $1,1,3,3$ \\
$\phi_{\mathrm{rec}}$ & $0,2,0,2$ \\
\hline
\end{tabular}




\section{CANCO}

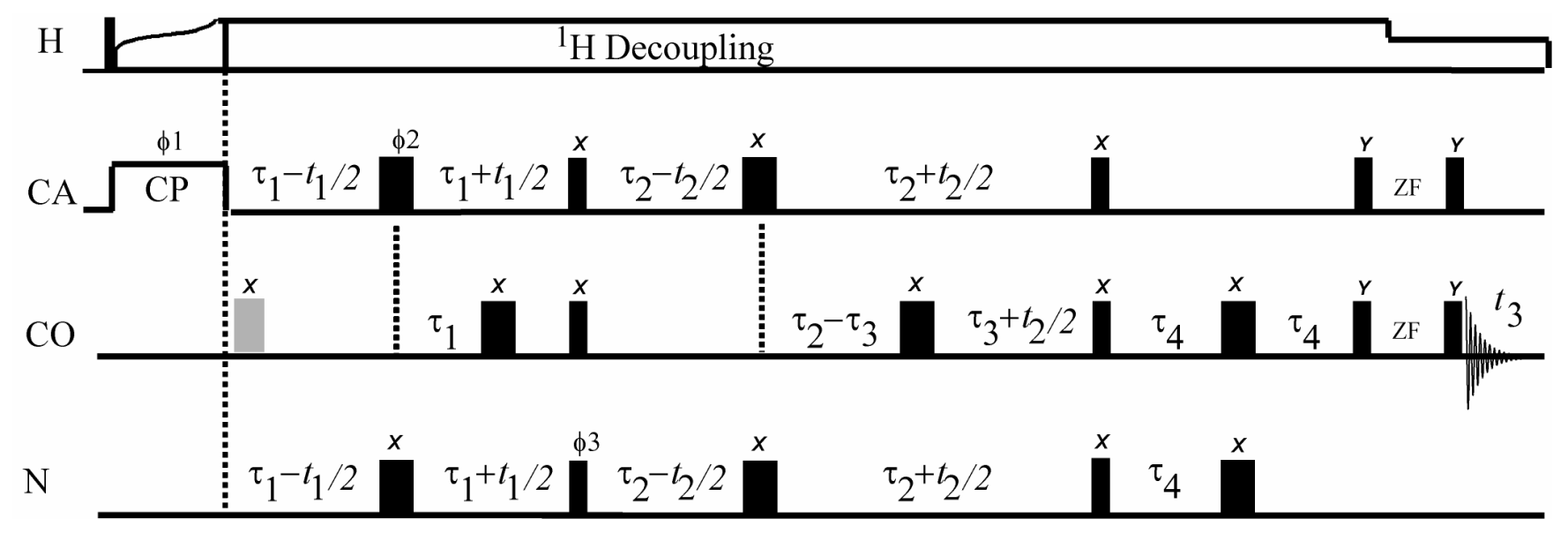

Off-Resonance Compensation: An additional $\pi$ pulse on the passive spin (shown as the grey, shaded pulse) can again be incorporated to refocus off-resonance effects should they be found to be significant.

Phase Cycle: Again, only a minimal phase cycle is necessary with the first ${ }^{13} \mathrm{C} \pi$ pulse $\left(\phi_{2}\right)$ cycled for $+/-1$. In the table below the phase of the rf is given as integer multiples of $90^{\circ}$ (i.e., $0=$ "x", $1=$ "y", $2=$ "-x", $3=$ "-y") and the list repeats once the end of the line has been reached.

3D pure phase spectra are obtained using the method of States (States, D. J.; Haberkorn, R. A.; Ruben, D. J. J. Magn. Reson. 1982, 48, 286-292) by incrementing $\phi_{1}$ by $90^{\circ}$ and $\phi_{3}$ by $90^{\circ}$ for $\mathrm{t}_{1}$ and $\mathrm{t}_{2}$, respectively.

\begin{tabular}{|c|l|}
\hline Phase & Cycle \\
\hline$\phi_{1}$ & 0 \\
$\phi_{2}$ & $0,1,2,3$ \\
$\phi_{3}$ & 0 \\
$\phi_{\mathrm{rec}}$ & $0,2,0,2$ \\
\hline
\end{tabular}




\section{$T_{2}$ vs. MAS Rate and Decoupling Power}

How well the J-MAS experiments work depends strongly on relaxation rates during the echo transfer periods (often denoted $\mathrm{T}_{2}{ }^{\prime}$ ). We measured $\mathrm{T}_{2}{ }^{\prime}$ over a range of MAS rates and proton decoupling powers for GB1 at $9.4 \mathrm{~T}$ using a simple $\tau$ $\pi$ (selective) $-\tau$ dephasing period. The echo intensity fall-off was then fit to an exponential decay in $\tau$ (typically using 6-7 time points). In each case, SPINAL 64 decoupling was used and optimized for maximum $\mathrm{T}_{2}$ ' on the alpha carbon and then used without further optimization for the carbonyl and nitrogen decays. $3.2 \mathrm{msec}$ and $420 \mu \mathrm{sec}$ r-SNOB pulses were used for the selective alpha and carbonyl pulses, respectively. The $3.2 \mathrm{msec}$ alpha pulse was found to be necessary to provide consistent echo line shapes without distortion from beta couplings. In all cases, we did not try to measure echo decay rates for individual sites, but rather an average rate for the spectral window. There are a number of experimental conditions with reduced MAS rates and decoupling powers where the relaxation rates are still quite favorable. For example, $18 \mathrm{kHz}$ MAS and $125 \mathrm{kHz}$ decoupling (routine for $3.2 \mathrm{~mm}$ probes) has very good relaxation properties, essentially the same as the $25 \mathrm{kHz}$ MAS and $150 \mathrm{kHz}$ decoupling we used, while $9 \mathrm{kHz}$ MAS and $100 \mathrm{kHz}$ decoupling (routine for $4 \mathrm{~mm}$ probes) is still quite respectable.

$\mathrm{T}_{2}^{\prime}(\mathrm{ms})$ at MAS $9 \mathrm{kHz}$

\begin{tabular}{c|ccc}
\hline${ }^{1} \boldsymbol{H}$ Dec. & $\boldsymbol{N}$ & $\boldsymbol{C O}$ & $\boldsymbol{C A}$ \\
\hline $\mathbf{7 5}$ & 29 & 22 & 13 \\
$\mathbf{1 0 0}$ & 55 & 33 & 20 \\
$\mathbf{1 2 5}$ & 51 & 36 & 21 \\
\hline $\mathbf{1 5 0}$ & 76 & 40 & 22 \\
\hline
\end{tabular}

$\mathrm{T}_{2}{ }^{\prime}(\mathrm{ms})$ at MAS $18 \mathrm{kHz}$

\begin{tabular}{c|ccc}
\hline${ }^{1} \boldsymbol{H}$ Dec. & $\boldsymbol{N}$ & $\mathbf{C O}$ & $\boldsymbol{C A}$ \\
\hline $\mathbf{7 5}$ & 28 & 30 & 9 \\
$\mathbf{1 0 0}$ & 68 & 62 & 25 \\
$\mathbf{1 2 5}$ & 96 & 69 & 33 \\
\hline $\mathbf{1 5 0}$ & 101 & 84 & 30 \\
\hline
\end{tabular}

$\mathrm{T}_{2}{ }^{\prime}(\mathrm{ms})$ at MAS $25 \mathrm{kHz}$

\begin{tabular}{c|ccc}
\hline${ }^{1} \boldsymbol{H}$ Dec. & $\boldsymbol{N}$ & $\boldsymbol{C O}$ & $\boldsymbol{C A}$ \\
\hline $\mathbf{7 5}$ & 24 & 28 & 8 \\
$\mathbf{1 0 0}$ & 43 & 44 & 16 \\
$\mathbf{1 2 5}$ & 90 & 83 & 32 \\
\hline $\mathbf{1 5 0}$ & 104 & 96 & 36 \\
\hline
\end{tabular}



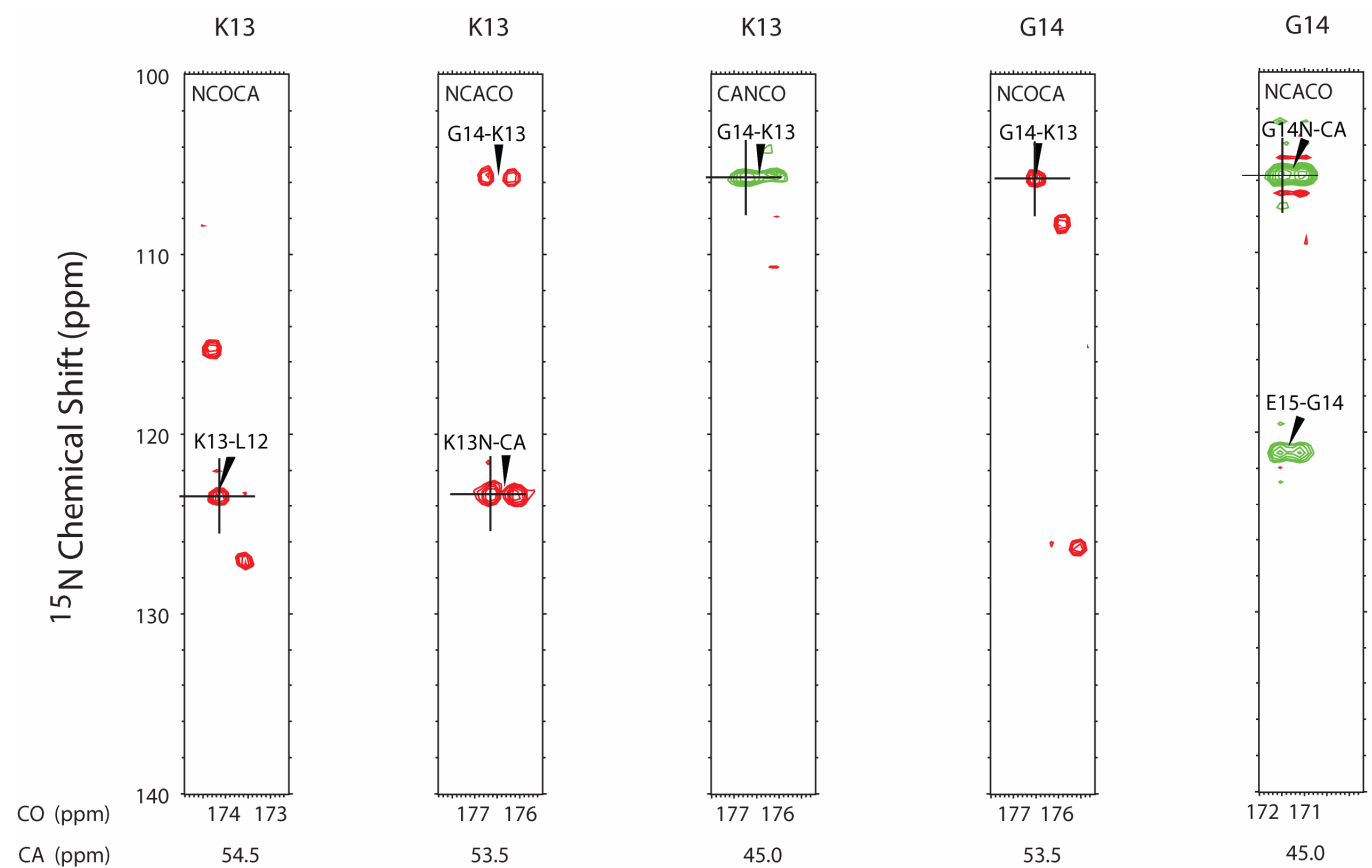

G14
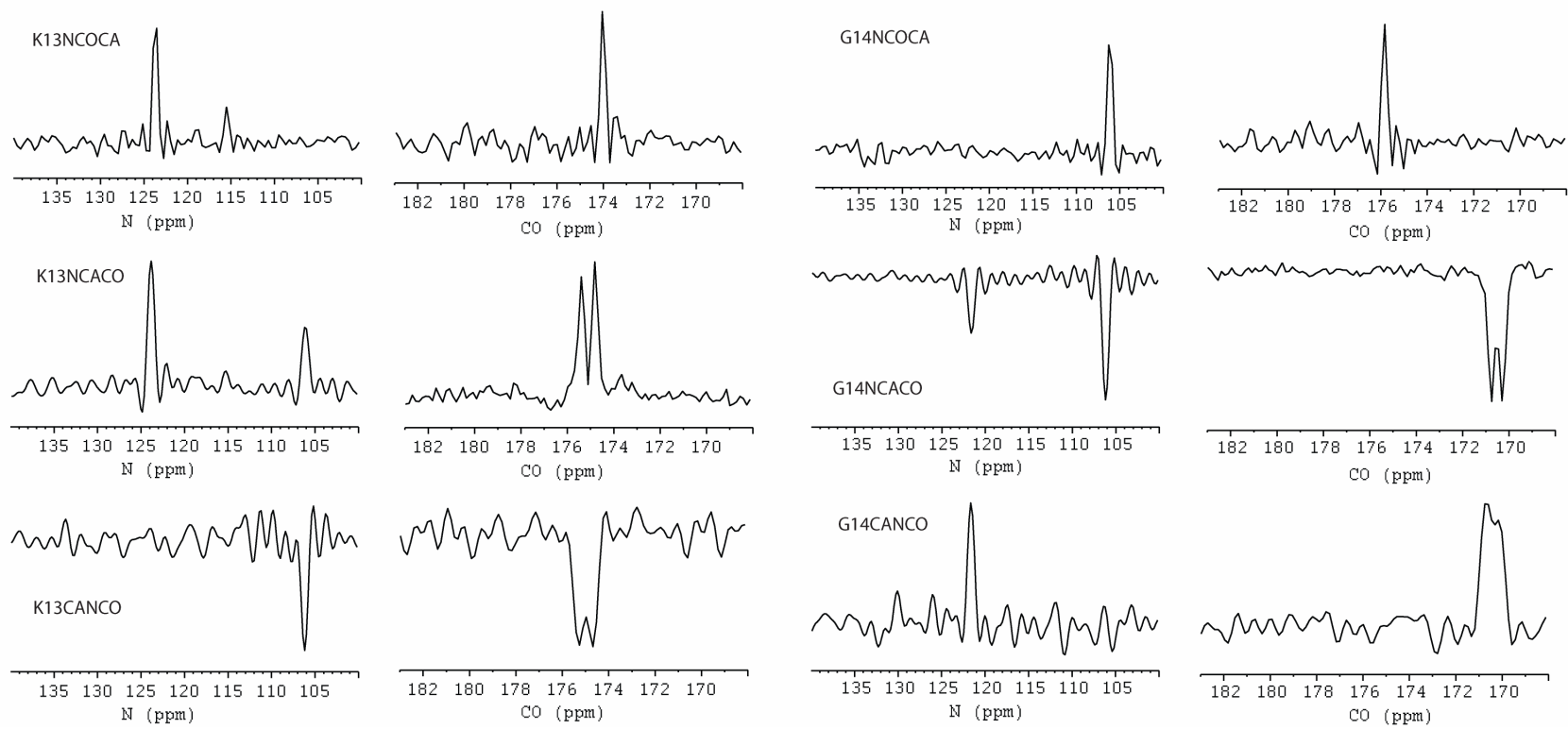

1D slices are shown for several of the strip plots from Figure 2. The majority of these slices show signal-to-noise ratios over 20:1. Taking 4:1 as the limit where it is still possible to consistently discriminate peaks from noise, we anticipate that assigning proteins up to 5 times larger than the $6 \mathrm{kDa}$ GB1 should be feasible even at $9.4 \mathrm{~T}$, while moving to higher field will extend this even further. In practice, the upper end of this range may require additional signal averaging, but should still be accessible within reasonable experimental times. 


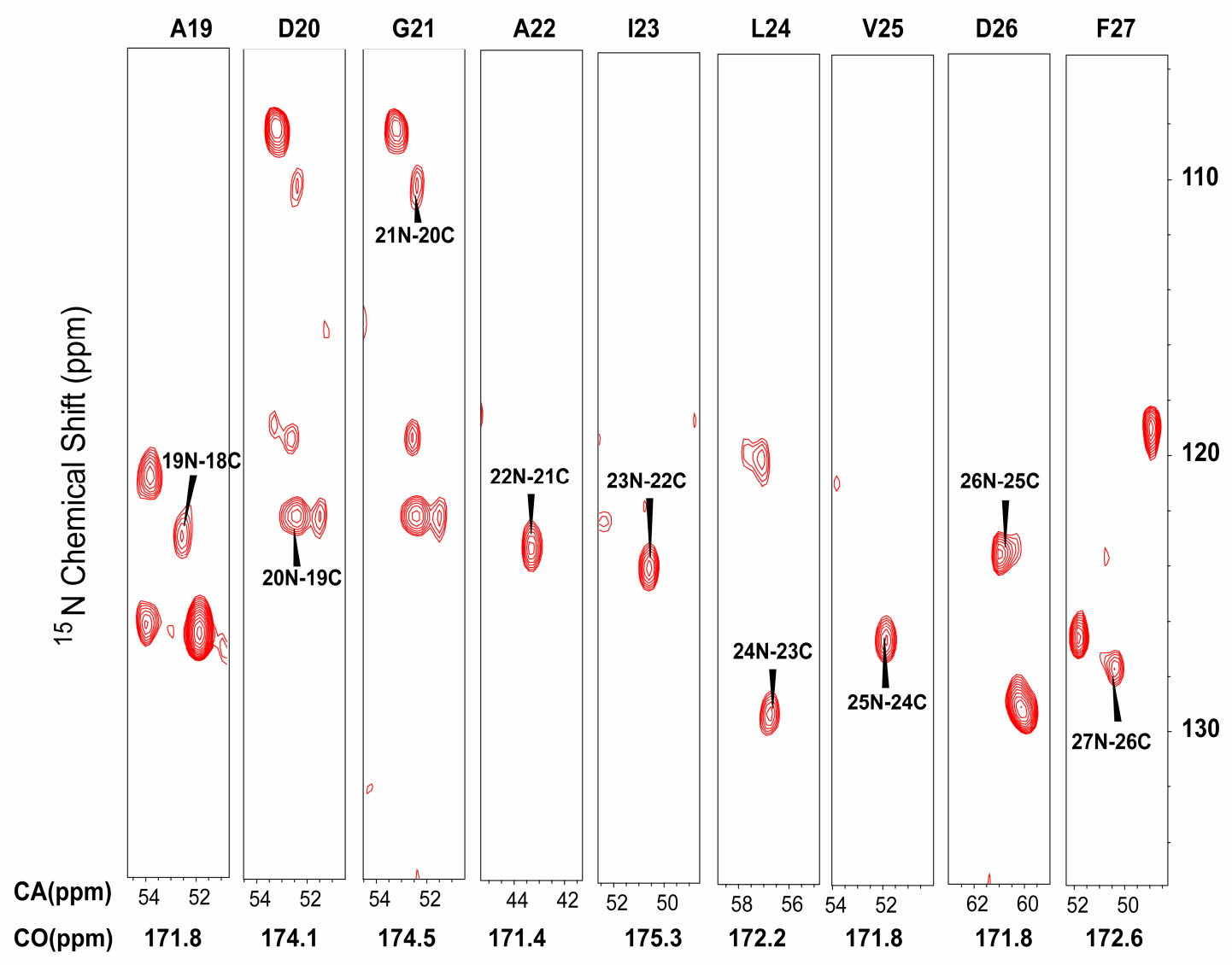

Figure S1: Strip plot of the 3D NCOCA spectrum of reassembled thioredoxin. The spectra were acquired at $14.1 \mathrm{~T}$ on a Varian InfinityPlus spectrometer outfitted with the triple-resonance $3.2 \mathrm{~mm}$ T3 probe. Acquisition parameters: 128 scans, 833 complex points in $t_{3}$ (total acquisition time $16 \mathrm{~ms}$ ), 40 real points in $t_{1}$ and $t_{2}$ (total acquisition time $11.9 \mathrm{~ms}$ ). Recycle delay: $2 \mathrm{~s}$; total experiment time: 5 days. Phase sensitive detection in $t_{1}$ and $t_{2}$ using TPPI. MAS frequency of $14.705 \mathrm{kHz}$. 90, 83 and $50 \mathrm{kHz}{ }^{1} \mathrm{H}$ TPPM decoupling was employed during the J-evolution, the acquisition and the Z-filter periods, respectively. $\tau_{1}=\tau_{2}=11.5 \mathrm{~ms} ; \tau_{3}=\tau_{4}=2.7 \mathrm{~ms}$. 170 and $374 \mu$ selective rSNOB pulses were used for the carbonyl and aliphatic regions, respectively. The spectra were processed with cosine apodization in $t_{1}$ and $t_{3}$ dimension, and with 60-degree shifted sinebell apodization in $t_{2}$ dimension; linear prediction to twice the number of points was applied in the $t_{1}$ and $t_{2}$ dimensions.

TRX prepared as described in:

Marulanda, D.; Tasayco, M. L.; McDermott, A.; Cataldi, M.; Arriaran, V.; Polenova, T. J Am Chem Soc 2004, 126, 16608-16620. 


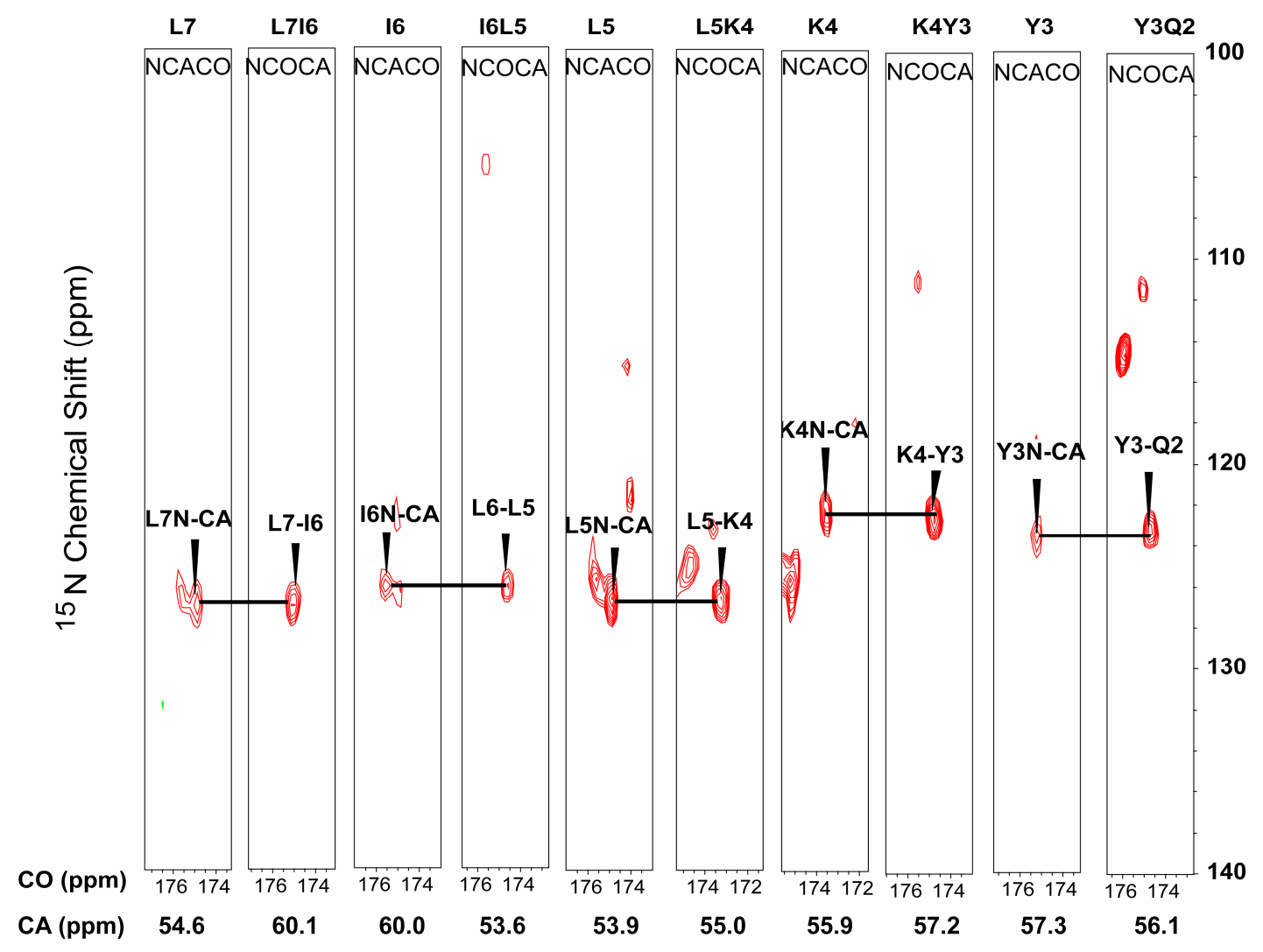

Figure S2: Strip plot of the 3D NCOCA and NCACO spectra of GB1. The spectra were acquired at $14.1 \mathrm{~T}$ on a Varian InfinityPlus spectrometer outfitted with the tripleresonance $3.2 \mathrm{~mm}$ T3 probe. Acquisition parameters (NCACO): 172 scans, 833 complex points in $t_{3}$ (total acquisition time $16 \mathrm{~ms}$ ), 25 and 19 real points in $t_{1}$ and $t_{2}$ respectively (total acquisition time in $t_{1}$ and $t_{2} 3.4$ and 1.48 ms respectively). Recycle delay: $2 \mathrm{~s}$; total experiment time: 45.3 hours. Acquisition parameters (NCOCA): 48 scans, 833 complex points in $t_{3}$ (total acquisition time $16 \mathrm{~ms}$ ), 38 real points in $t_{1}$ and $t_{2}$ (total acquisition time in $t_{1}$ and $t_{2} 5.2 \mathrm{~ms}$ ). Recycle delay: $2 \mathrm{~s}$; total experiment time: 40.3 hours. The following parameters apply to both experiments. Phase sensitive detection in $t_{1}$ and $t_{2}$ using TPPI. MAS frequency of $14.705 \mathrm{kHz} .90,83$ and $50 \mathrm{kHz}{ }^{1} \mathrm{H}$ TPPM decoupling was employed during the J-evolution, the acquisition and the Z-filter periods, respectively. For NCOCA, $\tau_{1}=\tau_{2}=11.5 \mathrm{~ms} ; \tau_{3}=\tau_{4}=2.7 \mathrm{~ms}$. For NCACO, $\tau_{1}=11.2 \mathrm{~ms}$; $\tau_{2}=4.4 \mathrm{~ms} ; \tau_{3}=\tau_{4}=2.7 \mathrm{~ms}$. For NCOCA, 170 and $374 \mu \mathrm{s}$ selective rSNOB pulses were used for the carbonyl and aliphatic regions, respectively. For NCACO, 442 and $374 \mu \mathrm{s}$ selective rSNOB pulses were used for the carbonyl and aliphatic regions, respectively. The spectra were processed with cosine apodization in $t_{1}$ and $t_{3}$ dimension, and with 60degree shifted sinebell apodization in $t_{2}$ dimension; linear prediction to twice the number of points was applied in the $t_{1}$ and $t_{2}$ dimensions.

GB1 prepared as described in:

Franks, W. T.; Zhou, D. H.; Wylie, B. J.; Money, B. G.; Graesser, D. T.; Frericks, H. L.; Sahota, G.; Rienstra, C. M. J Am Chem Soc 2005, 127, 12291-12305. 